\title{
S-17-5 6R-Tetrahydrobiopterin Biosynthesis: Structures of Rat and Human Sepiapterin Reductase
}

\author{
T.Nagatsu, ${ }^{1}$ H.IChinose, ${ }^{1}$ S.Katoh, ${ }^{2}$ K.FuJita, ${ }^{1}$ \\ and K.TITANI ${ }^{1}$
}

\begin{abstract}
${ }^{1}$ Institute for Comprehensive Medical Science, School of Medicine, Fujita Health University, Toyoake, Aichi 470-11 (Japan)

${ }^{2}$ Department of Biochemistry, School of Dentistry, Meikai University, Sakado, Saitama 350-02 (Japan)
\end{abstract}

I. INTRODUCTION

$(6 R)-2$-Amino-6-(L-erythro-1,2-dihydroxypropy 1) - 5, 6, 7,8tetrahydro-4 $(3 \mathrm{H})$-pteridinone (tetrahydrobiopterin, BPH4) is an essential cofactor for aromatic amino acid monooxygenases, phenylalanine, tyrosine, and tryptophan hydroxylases [1,2]. Tyrosine and tryptophan hydroxylases are the rate-limiting enzymes in the biosynthesis of monoamine neurotransmitters, i.e., catecholamines (dopamine, noradrenaline, and adrenaline) and serotonin, and their activities are regulated by several factors. Animals synthesize BPH4 in vivo from GPT through several enzymatic reactions. The concentration of $\mathrm{BPH} 4$ within the monoaminergic neurons and the adrenal medullary cells is thought to be one of the regulatory factors.

II. BPH4 BIOSYNTHESIS AND SEPIAPTERIN REDUCTASE (SPR)

Sepiapterin reductase $\left(7,8\right.$-dihydrobiopterin: NADP ${ }^{+}$ oxidoreductase, EC 1.1.1.153, SPR) [3] catalyzes the final step of $\mathrm{BPH} 4$ biosynthesis, and is thought to play an important role for the biosynthesis [4].

SPR shows a high specificity against a few carbonyl derivatives of pteridines. However, the enzyme can catalyze the NADPHdependent reduction of various carbonyl substances other than pteridines, and is considered to be one of the 'aldo-keto reductases', which have a broad specificity for endogenous and exogenous carbonyl compounds. It is important to clarify the complete structure of the enzyme molecule to analyze the complicated reaction mechanism of the enzyme. The enzyme purified from rat erythrocytes consists of two identical subunits each with an $\mathrm{Mr}$ of about 28,000. We have determined primary structures of rat SPR[5] and human SPR[6] by protein analysis and by CDNA cloning. We have found homology among human SPR, human carbonyl reductase, and human dehydrogenases.

III. STRUCTURE OF RAT SPR

Citron et al.[7] recently reported a partial nucleotide sequence of rat SPR. We determined $96 \%$ of the amino acid sequence of rat SPR including the $\mathrm{N}$-terminal structure by protein analysis [5]. The complete amino acid sequence of the mature form of rat SPR was provided by these two reports $[5,7]$.

We purified rat SPR from erythrocytes by the method of sueoka and Katoh [3], i.e., ammonium sulfate, hydroxyapatite, Matrix Red $A$, and isoelectric focusing. The purified SPR was reduced with dithiothreitol and carboxymethylated with monoiodoacetamide. The 
S-carboxyamidomethylated-protein was enzymatically and chemically cleaved. For enzymatic cleavage, Achromobacter protease I, TPCKtrypsin and $\alpha$-chymotrypsin were used. Chemical cleavage was carried out by cyanogen bromide and BNPS-skatole.

Peptides were separated by reversed-phase HPLC. Sequence analysis was performed with an Applied Biosystems Model $470 \mathrm{~A}$ Protein Sequencer on line connected to a 120A PTH analyzer.

The sequencer analysis could not identify the N-terminal sequence of the enzyme. Digestion of a N-blocked peptide was carried out with acyl amino acid releasing enzyme, and the analysis of the peptide with a sequencer yielded the sequence of N-acetyl-Met-Glu-Gly-Gly-Arg- .

We have sequenced $96.2 \%$ of the entire sequence of the mature form of the enzyme. Three important informations emerged from the present results. (1) Our results clearly demonstrated that the $\mathrm{N}$-terminus of the mature form of the enzyme is the methionine-1 and processed by N-acetylation. (2) Citron et al. [7] recently reported a partial nucleotide sequence of a CDNA encoding rat SPR, which lacks the $5^{\prime}$ sequence corresponding to the first 3-amino acids in the N-terminus of the protein. The amino acid sequence of rat SPR presented by us [7] completely agrees with the sequence derived from the rat CDNA by Citron et al.[5]. Comparison of the results with the predicted amino acid sequence provided the complete amino acid sequence of the mature form of the enzyme. (3) This also indicates that no proteolytic processing event occurs at the c-terminus of the enzyme, and also no covalent processing event other than N-acetylation, such as glycosylation or methylation, appears to occur in the entire molecule. The Mr of the subunit was calculated to be 28169.06 from this complete amino acid sequence of 262 residues including $\mathrm{N}$-terminal acetyl group. Therefore, the Mr of the native enzyme should be 56338.12

\section{STRUCTURE OF HUMAN SPR}

We [6] have cloned a full-length CDNA for human SPR from liver, and have determined the complete amino acid sequence of human SPR deduced from the nucleotide sequence of the cDNA. A clone (hSPR8-25) contained an entire coding region of human SPR. The nucleotide sequence of CDNA encoding human SPR and the deduced amino acid sequence are shown in Fig.1. The clone encoded a protein of 261 amino acids with a calculated Mr of 28047.

The predicted amino acid sequence of human SPR was compared to that of the rat enzyme $[5,7]$. The putative pterin binding site (Ala-Gly-Leu-Leu-Ser) suggested by Citron et al. [7] in rat SPR is shown on a solid line. As the amino acid sequence in human SPR corresponding to this sequence was Ala28-Ser-Leu-Leu-Ser32, the consensus sequence for a pterin-binding peptide should be Ala-XxxLeu-Leu-Ser. A NADPH binding consensus sequence, TL-L-G-G---G--L---L---L-L-D, spanning 28 amino acids from residues $13-40$ was well conserved between human and rat SPR.

SPR has a dehydrogenase activity, a reverse reaction against reductase, and also carbonyl reductase activity. Therefore, we compared the amino acid sequence of human SPR with those of human

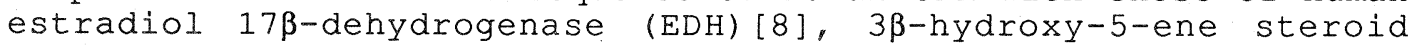
dehydrogenase (HSD) [9] and carbonyl reductase (CBR) [10]. They exhibit a striking homology with SPR, especially in their Nterminal region. The pattern of homology suggests that these molecules contain a similar core structure in the N-terminal portion containing the determinants for the enzymatic activity. 


$$
-20-10 \quad-1
$$

GCCGCCGGCGGAGAACAGGAGC

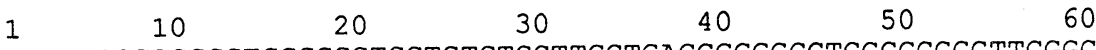
ATGGAGGGCGGGCTGGGGCGTGCTGTGTGCTTGCTGACCGGGGCCTCCCGCGGCTTCGGC $\begin{array}{llllllllllllllllllll}M & E & G & G & L & G & R & A & V & C & I & L & T & G & \underline{A} & \underline{S} & \underline{R} & \underline{G} & \underline{G} & \underline{G}\end{array}$

$\begin{array}{llllll}70 & 80 & 90 & 100 & 110 & 120\end{array}$
CGGACGCTGGCCCCGCTCCTGGCCTCGCTGCTGTCGCCCGGCTCCGTGCTTGTCCTTAGC R I I $\underline{\mathrm{A}} \underline{\mathrm{P}} \underline{\mathrm{L}} \underline{\mathrm{A}} \underline{\mathrm{S}} \underline{\mathrm{L}} \underline{\mathrm{S}} \underline{\mathrm{P}} \underline{\mathrm{G}} \underline{\mathrm{V}} \underline{\mathrm{V}} \underline{\mathrm{V}} \underline{\mathrm{L}}$

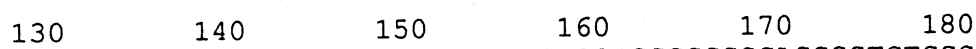
GCCCGCAACGACGAGGCACTGCGCCAGCTGGAGGCCGAGCTGGGCGCCGAGCGGTCTGGC $\begin{array}{llllllllllllllllllll}A & R & N & D & E & A & L & R & Q & L & E & A & E & L & G & A & E & R & S & G\end{array}$

$190 \quad 200 \quad 210 \quad 220 \quad 230 \quad 240$
CTGCGCGTGGTGCGGGTGCCCGCCGACCTGGGCGCCGAGGCCGGCTTGCAGCAGCTGCTC $\begin{array}{llllllllllllllllllll}I & R & V & V & R & V & P & A & D & L & G & A & E & A & G & L & Q & Q & I & I\end{array}$

$250 \quad 260 \quad 270 \quad 280 \quad 290 \quad 300$
GGCGCCCTGCGCGAGCTCCCCCGGCCCAAGGGGCTGCAGCGACTGCTGCTTATCAACAAC $\begin{array}{lllllllllllllllllllll}G & A & L & R & E & L & P & R & P & K & G & L & Q & R & L & I & L & I & N & N\end{array}$

$\begin{array}{lllll}310 & 320 & 330 & 340 & 350\end{array}$
GCGGGCTCTCTTGGGGATGTGTCCAAAGGCTTCGTGGACCTGAGTGACTCCACTCAAGTG $\begin{array}{llllllllllllllllllll}A & G & S & L & G & D & V & S & K & G & F & V & D & L & S & D & S & T & Q & V\end{array}$

$\begin{array}{lllll}370 & 380 & 390 & 400 & 410\end{array}$
AACAACTACTGGGCACTGAACTTGACCTCCATGCTCTGCCTGACTTCCAGCGTCCTGAAG $\begin{array}{llllllllllllllllllll}N & N & Y & W & A & L & N & L & T & S & M & L & C & L & T & S & S & V & L & K\end{array}$

$\begin{array}{llllll}430 & 440 & 450 & 460 & 470 & 480\end{array}$
GCCTTCCCGGACAGTCCTGGCCTCAACAGAACCGTGGTTAACATCTCGTCCCTCTGTGCC $\begin{array}{llllllllllllllllllll}\mathrm{A} & \mathrm{F} & \mathrm{P} & \mathrm{D} & \mathrm{S} & \mathrm{P} & \mathrm{G} & \mathrm{L} & \mathrm{N} & \mathrm{R} & \mathrm{T} & \mathrm{V} & \mathrm{V} & \mathrm{N} & \mathrm{I} & \mathrm{S} & \mathrm{S} & \mathrm{L} & \mathrm{C} & \mathrm{A}\end{array}$

$490 \quad 500 \quad 510 \quad 520 \quad 530 \quad 540$
CTGCAACCTTTCAAAGGCTGGGCGCTGTACTGTGCAGGAAAGGCTGCTCGTGATATGCTG $\begin{array}{llllllllllllllllllll}\mathrm{L} & Q & \mathrm{P} & \mathrm{F} & \mathrm{K} & \mathrm{G} & \mathrm{W} & \mathrm{A} & \mathrm{L} & \mathrm{Y} & \mathrm{C} & \mathrm{A} & \mathrm{G} & \mathrm{K} & \mathrm{A} & \mathrm{A} & \mathrm{R} & \mathrm{D} & \mathrm{M} & \mathrm{I}\end{array}$

$550 \quad 560 \quad 570 \quad 580 \quad 590 \quad 600$
TTCCAGGTCCTGGCGCTGGAGGAACCTAATGTGAGGGTGCTGAACTATGCCCCAGGTCCT $\begin{array}{llllllllllllllllllll}\mathrm{F} & Q & \mathrm{~V} & \mathrm{~L} & \mathrm{~A} & \mathrm{~L} & \mathrm{E} & \mathrm{E} & \mathrm{P} & \mathrm{N} & \mathrm{V} & \mathrm{R} & \mathrm{V} & \mathrm{L} & \mathrm{N} & \mathrm{Y} & \mathrm{A} & \mathrm{P} & \mathrm{G} & \mathrm{P}\end{array}$ $\begin{array}{llllll}610 & 620 & 630 & 640 & 650 & 660\end{array}$ CTGGACACAGACATGCAGCAGTTGGCCCGGGAGACCTCCGTGGACCCAGACATGCGAAAA $\begin{array}{llllllllllllllllllll}L & D & T & D & M & Q & Q & I & A & R & E & T & S & V & D & P & D & M & R & K\end{array}$

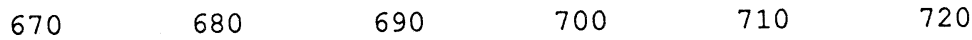
GGGCTGCAGGAGCTGAAGGCAAAGGGGAAGCTGGTGGATTGCAAGGTGTCAGCCCAGAAA $\begin{array}{llllllllllllllllllll}G & L & Q & E & L & K & A & K & G & K & L & V & D & C & K & V & S & A & Q & K\end{array}$

$\begin{array}{lllll}730 & 740 & 750 & 760 & 770\end{array}$
CTGCTGAGCTTACTGGAAAAGGACGAGTTCAAGTCTGGAGCCCACGTGGACTTCTATGAC $\begin{array}{llllllllllllllllllll}L & L & S & L & L & E & K & D & E & E & K & S & G & A & H & V & D & F & Y & D\end{array}$

$790 \quad 800 \quad 810$

AAATAAGCCCATGTTTTTGGCTTCCTGAACC $\mathrm{K}$ *

Fig.1. The nucleotide sequence encoding human sepiapterin reductase ( $S P R$ ) and the deduced amino acid sequence. The consensus sequence for a pterin-binding site is shown on a solid line, and that of a NADPH-binding site is shown on a dotted line. 
ACKNOWLEDGEMENTS

This work was supported by a Grant-in-Aid for Scientific Research from the Ministry of Education, Science and Culture, Japan. We wish to thank Dr. Y.Ebina (The University of Tokushima) for providing a human liver library.

\section{REFERENCES}

[1] Kaufman, S., and Fisher, D.B. (1974): Pterin-requiring aromatic amino acid hydroxylases, in Molecular Mechanism of Oxygen Activation, ed. by Hayaishi, 0., Academic Press, New York, pp. 285-369.

[2] Nagatsu, T. (1985): Biopterin cofactor and monoaminesynthesizing monooxygenases, ed. by Osborn, N. N., Pergamon Press, Oxford, pp. 325-340.

[3] Sueoka, T., and Katoh, S. (1982): Purification and characterization of sepiapterin reductase from rat erythrocytes. Biochim. Biophys. Acta, 717, 265-271.

[4] Levine, R.A., Kapatos, G., Kaufman, S., and Milstien, S. (1990): Immunological evidence for the requirement of sepiapterin reductase for tetrahydrobiopterin biosynthesis in brain. J. Neurochem., 54, 1218-1224.

[5] Oyama, R., Katoh, S., Sueoka, T., Suzuki, M., Ichinose, H., Nagatsu, T., and Titani, K. (1990): The complete amino acid sequence of the mature form of rat sepiapterin reductase. Biochem. Biophys. Res. Commun., 173, 627-631.

[6] Ichinose, H., Katoh, S., Sueoka, T., Titani, K., Fujita, K., and Nagatsu, T. (1991): Cloning and sequencing of CDNA encoding human sepiapterin reductase - an enzyme involved in tetrahydrobiopterin biosynthesis. Biochem. Biophys. Res. Commun., in press.

[7] Citron, B.A., Milstien, S., Gutierrez, J.C., Levine, R.A., Yanak, B.L., and Kaufman, S. (1990): Isolation and expression of rat liver sepiapterin reductase cDNA. Proc. Natl. Acad. Sci. USA, 87, 6436-6440.

[8] Luu, V.T., Labrie, C., Zhao, H.F., Couet, J., Lachance, Y., Simard, J., Lablance, G., Cote, J., Berube, D., Cagne, R., and Labrie, F. (1989): Characterization of CDNA for human estradiol 17 $\beta$-dehydrogenase and assignment of the gene to chromosome 17: evidence of two mRNA species with distinct $5^{\prime}-$ termini in human placenta. Mol. Endocrinol., 3, 1301-1309.

[9] Luu, V.T., Lachance, Y., Labrie, C., Leblance, G., Thomas, J.L., Strickler, R.C., and Labrie, F. (1989): Full length CDNA structure and deduced amino acid sequence of human $3 \beta-$ hydroxy-5-ene steroid dehydrogenase. Mol. Endocrinol., 3, 1310-1312.

[10] Forrest, G.L., Akman,M S., Krutzik, S., Paxton, R.J., Sparkes, R.S., Doroshow, J., Felsted, R.L., Glover, C.J.', Mohandas, T., and Bachur, N.R. (1990): Induction of a human carbonyl reductase gene located on chromosome 21 . Biochim. Biophys. Acta, 1048, 149-155. 